\title{
EFFECT OF FEEDBACK ON PERFORMANCE IN THE LANE-CHANGE TEST
}

\author{
Vera Berthon-Donk, Marc Grosjean, \& Gerhard Rinkenauer \\ Leibniz Research Centre for Working Environment and Human Factors \\ Dortmund, Germany \\ Email:donk@ifado.de
}

\begin{abstract}
Summary: The Lane-Change Test (LCT) is an easy-to-use methodological tool that has proven useful for researching dual-task driving situations. This paper examines the effect of feedback on LCT performance. Feedback is important for maintaining the focus of attention on the primary (driving) task and providing motivation for learning. An experiment was conducted in which two driver groups performed the LCT with or without end-of-block summary feedback. Results showed that the presence of feedback significantly improved performance, as revealed by lower overall means and lower standard deviations (with practice) of lateral deviation values. We conclude that feedback can have a positive effect on performance in the LCT and, therefore, it may be critical to include such feedback when using this, as well as similar tasks, to investigate dual-task driving situations.
\end{abstract}

\section{INTRODUCTION}

The Lane-Change Test (LCT; Mattes, 2003) is an easy-to-use tool to study dual-task driving situations. The LCT is a PC-based desktop application which provides a standardized driving task that allows one to measure in-vehicle secondary-task demands. The test has become an ISO standard (ISO 26022, 2010) and represents a simplified driving environment in which lane changes need to be performed in response to lane-change signs. The amount of distraction is assessed by changes in performance on lane maneuvers that are executed while dealing with a secondary task. The LCT has also proven to be a valuable tool for studying learning effects in dual-task driving situations (Chisholm, Caird, \& Lockhart, 2008; Petzoldt, Bär, Ihle, \& Krems, 2011; Shinar, Tractinsky, \& Compton, 2005).

Although the LCT seems to be a useful tool, some issues remain to be clarified. If we consider real-life driving, it might appear obvious that driving receives far more priority than any secondary task so that accidents and collisions are avoided. In a simulated driving environment, however, shifts in prioritization might take place from the primary (driving) task to the secondary task: As there is no real risk, participants might focus more on the secondary than on the primary task. Consistent with this view, Levy and Pashler (2008) showed that even when participants in a simulated dual-task driving situation were explicitly told to ignore the secondary task and give maximum priority to the driving task, they would often nonetheless respond to stimuli from the secondary task and thereby cause dual-task interference. Related to these findings, Brumby, Salvucci, and Howes (2007) used feedback as a method to emphasize the focus towards either the primary or the secondary task in a simulated dual-task driving situation, while studying strategic dual-task trade-offs. Their findings showed that feedback could indeed be used for changing task priority (see also Donmez, Boyle, \& Lee, 2006). 
Feedback is also important for learning as it provides motivation (Vollmeyer \& Rheinberg, 2000) and guidance (Salmoni, Schmidt, \& Walter, 1984). For example, summary knowledge of results (SKR) is a form of feedback that provides information about goal achievement only after the last trial has been completed. It has been proven to help in-depth learning without causing a lot of variability between trials (Schmidt, Lange, \& Young, 1990). SKR may therefore be a good type of feedback for the LCT, as it shouldn't interfere with lane changes. SKR also provides information that could increase motivation while performing the LCT, which should result in better driving performance. We therefore sought to examine the effect of SKR on LCT performance. In particular, we expected participants with feedback, due to increased levels of motivation and guidance provided by SKR, to perform more accurate lane-change maneuvers. We furthermore expected the presence of feedback to lead to a reduction in the amount of performance variability (Schmidt et al., 1990).

\section{METHOD}

\section{Participants}

Forty individuals ( 20 men, 20 women, mean age $=24.0$ years, age range $=21-29$ years $)$ participated in the study. All participants held a valid driver license, drove on average $14615 \mathrm{~km}$ per year $(\mathrm{SD}=6003 \mathrm{~km})$, were naïve to the purpose of the study, had normal or corrected-tonormal vision, and were paid for participation.

\section{Material \& Tasks}

Lane-Change Test. Participants were presented with a simulated roadway consisting of a straight 3-lane road without other traffic and were instructed to perform as fast and accurately as possible lane changes to the left or the right of 1 or 2 lanes. Each track contained 18 lane-change signs along the road that indicated the type of lane change (direction and number of lanes) to be performed. Each sign was initially blank and only became visible $40 \mathrm{~m}$ before reaching it, at which point lane-change information is provided. Driving speed was held constant at $60 \mathrm{~km} / \mathrm{h}$. A "track" was considered as an experimental "block", and therefore those terms will be used interchangeably.

The hardware for the LCT consisted of an IBM compatible PC with a Logitech ${ }^{\circledR}$ steering wheel and foot pedals. The driving image was projected onto a projection screen. The resolution of the projected image was set to $1024 \times 768$ pixels and subtended a visual angle of $33.4^{\circ} \times 43.4^{\circ}$. The participants were seated in a height-adjustable office chair, at a viewing distance of $2 \mathrm{~m}$ in front of the middle of the projection screen. Driving data were recorded with a frequency of $100 \mathrm{~Hz}$ and contained the following variables: Trial number, time, reaction time, $x-$, and $y$-coordinates of the actual position of the virtual vehicle, steering wheel angle and heading angle.

Rating Scale of Mental Effort (RSME) \& NASA Task Load Index (NASA-TLX). For a subjective measure of workload after each track, we employed the RSME (Zijlstra \& Van Doorn, 1985). This simple one-dimensional ratio scale allows individuals to rate their mental effort from 0 to 150. The NASA-TLX (Hart \& Staveland, 1988) was filled out at the end of the experiment. This multidimensional questionnaire is intended to assess mental workload experienced while 
performing a task according to six dimensions: Mental demand, physical demand, temporal demand, performance, effort, and frustration.

\section{Design \& Procedure}

A mixed-design with one between-participants factor (feedback condition: feedback, no feedback) and one within-participants factor (block: 1-10) was used. Participants were randomly assigned to the feedback or no-feedback condition in a counter-balanced fashion. They first signed a consent form and filled out some demographic questionnaires. Then they received written instructions describing the task at hand, in which it was emphasized that good performance included lane-change speed and accuracy, as well as lane keeping in the middle of the lane. Each participant then drove at least 1 practice block in which his/her overall lanechange and lane-keeping performance was summarized with the mean deviation (MDEV) value. MDEV is a measure of lateral control that is obtained by comparing the actual driving trajectory of the participant to a reference trajectory (Mattes \& Hallén, 2009). In accordance with the ISOnorm, participants had to reach an MDEV value of less than $1.2 \mathrm{~m}$ (Mattes \& Hallén, 2009). If the participant didn't manage to reach this level, the practice block was repeated until this level was reached. Generally one practice block was enough to attain the criterion MDEV value. In all conditions, the participants received precise oral instructions from the experimenter on how to increase their performance, such as making lurching moves in the lane-change segments and concentrating on the car's outer dimensions while lane keeping. The participants then had to drive 10 experimental blocks. After each block, participants had to rate their mental effort on the RSME. Participants in the feedback condition would then receive feedback on their performance. Feedback consisted of the MDEV value, which was compared to a very good (0.3) or a very bad (2.5) value, and the number of signs they misinterpreted or missed (if any). The no-feedback group did not receive any feedback. At the end of the experiment, each participant had to fill out the NASA-TLX and rate their general mean subjective workload for the last 10 tracks. The entire experiment took approximately 60 minutes.

\section{RESULTS \& DISCUSSION}

Data from the practice blocks were discarded. Separate two-way (feedback condition $\mathrm{x}$ block) mixed-factor analyses of variance (ANOVAs) were conducted on the following measures: MDEV, standard deviation of lateral deviation (SDDEV), standard deviation of the steering wheel angle (SDSW), and RSME. Two different values were computed and analyzed separately for MDEV, SDDEV, and SDSW: One for lane change (starting with the visibility of the sign 40 $\mathrm{m}$ before the sign and ending $56 \mathrm{~m}$ after the sign) and one for lane keeping (starting $80 \mathrm{~m}$ before the following sign and ending $40 \mathrm{~m}$ before that sign). Trials in which participants had made an incorrect lane change or had deviated into another lane in lane-keeping segments were excluded from analysis, as they would otherwise inflate the lateral deviation values. A total of $0.33 \%$ of the trials was discarded. The effect of feedback condition on the NASA-TLX scores were analyzed with separate independent samples $t$ tests for each of the 6 items. Probability level for statistical significance was set to .05 and sphericity violations were corrected for with the Greenhouse-Geisser $\varepsilon$ (uncorrected degrees of freedom are provided). 
$M D E V$. The left panel in Figure 1 presents the results for MDEV as a function of block and feedback condition for lane-change segments. The ANOVA yielded a main effect of block $\left(F[9,342]=7.63, \eta_{\mathrm{p}}{ }^{2}=.17, p<.001\right)$, which reflects that MDEV decreased with practice. There was also a main effect of feedback condition $\left(F[1,38]=5.10, \eta_{\mathrm{p}}{ }^{2}=.12, p<.05\right)$ : The feedback group performed better lane changes, as reflected by lower MDEVs, than the no-feedback group. In the lane-keeping segments (not shown here), the main effect block did not reach significance and there was no main effect of feedback condition either, although a tendency was observed $\left(F[1,38]=3.20, \eta_{\mathrm{p}}{ }^{2}=.08, p=.08\right)$. Neither of the interactions (for lane change or lane keeping) were significant (both $p \mathrm{~s}>.31$ ).
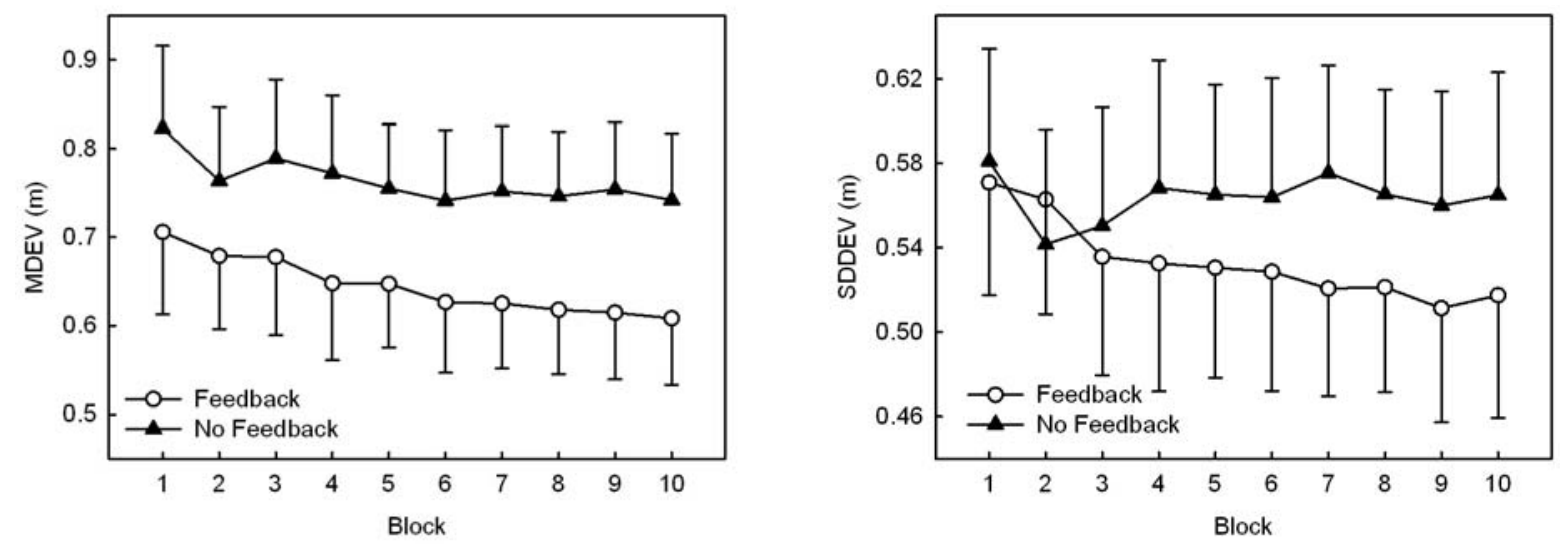

Figure 1. Mean lateral deviation (MDEV) (left) and standard deviation of lateral deviation (SDDEV) (right) for lane-change segments as a function of block (1-10) and feedback condition (feedback, no feedback) (Error bars correspond to half of the respective $95 \%$ (between-participants) confidence interval)

$S D D E V$. The right panel in figure 1 represents the results for SDDEV as a function of block and feedback condition for lane-change segments. There was no main effect of feedback $(p>.40)$ in the lane-change segments, but the interaction between block and feedback was significant $(F[9$, $\left.342]=2.30, \eta_{\mathrm{p}}{ }^{2}=.06, p<.05\right)$ : SDDEV values were initially similar for both feedback conditions and subsequently decreased with practice (block) for the feedback group only. As for the lane-keeping segments (not shown here), the feedback group $(\mathrm{M}=0.09 \mathrm{~m}$ ) showed less variability than the no-feedback $(\mathrm{M}=0.12 \mathrm{~m})$ group $\left(F[1,38]=6.13, \eta_{\mathrm{p}}{ }^{2}=.14, p<.05\right)$. No other significant main effects or interactions were observed (all $p \mathrm{~s}>.08$ ).

SDSW. Figure 2 shows SDSW for lane-change segments as a function of block and feedback condition. The ANOVA yielded a main effect of block $\left(F[9,342]=3.12, \eta_{\mathrm{p}}{ }^{2}=.08, p<.05\right)$ and a significant interaction between block and feedback condition $\left(F[9,342]=3.85, \eta_{\mathrm{p}}{ }^{2}=.09, p<\right.$ $.05)$. These effects reflect that both groups initially had similar SDSW values and that they subsequently decreased (before leveling off) for the no-feedback group only. As for the lanekeeping segments (not shown here), there was a main effect of block $\left(F[9,342]=7.28, \eta_{\mathrm{p}}{ }^{2}=.16\right.$, $p=.01)$ : SDSW decreased overall with practice from block $1(\mathrm{M}=1.00 \mathrm{deg})$ to block $10(\mathrm{M}=$ $0.64 \mathrm{deg})$. The block $\mathrm{x}$ feedback interaction was not significant for lane-keeping segments $(p>$ .17 ) and there was no main effect of feedback for either type of segment (both $p s>.14$ ). 


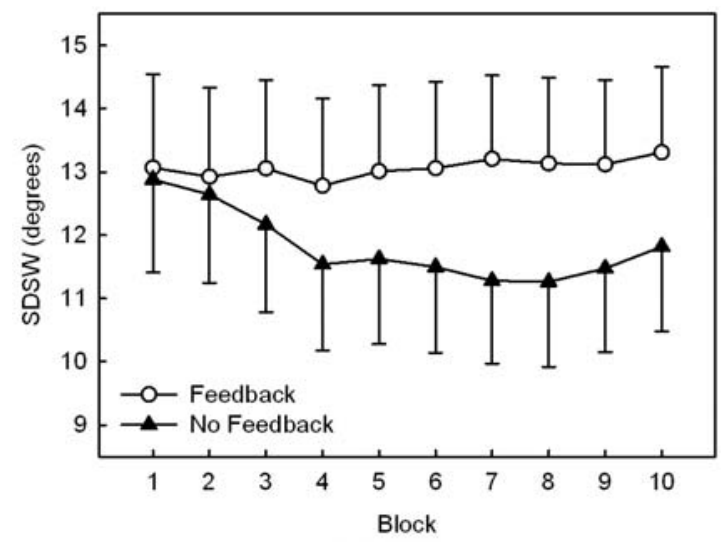

Figure 2. Standard deviation of steering wheel angle (SDSW) for lane-change segments as a function of block (1-10) and feedback condition (feedback, no feedback)

(Error bars correspond to half of the respective 95\% (between-participants) confidence interval)

RSME \& NASA-TLX. RMSE data showed no significant main effects or interactions (all ps > .23). As for the NASA-TLX, only the scores on the subscale "performance" showed a significant difference between the scores for feedback $(M=111.66)$ and no-feedback $(M=91.95)$ conditions $(t[38]=2.04, p<.05)$. This subscale describes the degree of success or satisfaction felt upon the performance or completion of a given task. A high score on this scale means that the participant rates his own performance as poor, a low score as good. Thus, these results suggest that the presence of feedback lead to more pessimistic subjective ratings.

\section{GENERAL DISCUSSION}

Taken together, the results of this experiment show that SKR feedback has an effect on driving performance in the LCT. Specifically, our results revealed that when such feedback is provided, participants are better (in terms of MDEV) at performing lane changes. The presence of feedback also led to a decrease in lateral-deviation variability (SDDEV) with practice for lane-change segments, and less variability overall for lane-keeping segments. Thus, feedback seems to have an effect on learning as well: The amount of variability decreased with practice in lane-change segments for the feedback group only. Lastly, feedback didn't have an effect on most of the subjective measures, except for the subjective measure of "performance" on which participants in the feedback group rated their own performance in a more pessimistic manner.

There was one apparently counter-intuitive result: SDSW in lane-change segments decreased with practice only for participants without feedback. In general, studies have shown that SKR leads to a decrease in performance variability, which our results concerning lateral deviation variability (SDDEV) confirmed. One account for the SDSW pattern found in the current study could be that a certain amount of steering-wheel movement variability is related to corrective steering movements. The SDSW results thus suggest that both groups initially performed a similar amount of corrective movements. However, only the feedback group continued to do so, thereby leading to a roughly constant level of steering-wheel variability, but better performance than the no-feedback group overall. This would be consistent with the idea that variability also reflects the ability to compensate for unintended deviations (Latash, Scholz, \& Schöner, 2002). 
Our study suggests that SKR provides an effective type of feedback to help increase driving performance. One may, however, speculate that Knowledge of Results (KR) on a trial-to-trial basis would be more effective than SKR. Although KR has been proven to have a beneficial effect on performance (Salmoni et al., 1984), it also leads to more performance variability and less in-depth learning (Schmidt \& Wulf, 1997). Given that steering maneuvers in the LCT are already associated with continuous visual and proprioceptive feedback, adding KR on a trial-totrial basis may actually cause distraction, and thereby be more harmful than beneficial. It should furthermore be noted that participants in this study didn't receive any information on how to increase performance. They received feedback on how their performance differed from an optimal performance, and therefore the current findings may only reflect motivational dynamics. Knowledge of performance (KP), such as providing participants not only with performance feedback but also with information on how to improve performance, is a well-researched way of providing procedural information to enhance in-depth learning (Ammons, 1956). It can be expected that when providing KP, performance on the LCT would increase even further.

From a theoretical perspective, our findings are consistent with literature on feedback according to which performance increases (Schmidt et al., 1990) due to guidance (Salmoni et al., 1984) and motivation (Vollmeyer \& Rheinberg, 2000). However, although the presence of SKR leads to better performance in the LCT, it is still unclear whether such feedback would actually help participants to focus primarily on the driving task instead of any additional tasks. Some evidence in favor of this idea comes from the study of Brumby et al. (2007) considered above. Recall that they were able to successfully shift the focus of attention with the use of feedback from one task to the other in a dual-task driving situation. Furthermore, Levy and Pashler (2008) found that, in a simulated dual-task driving environment without feedback, participants do not always place priority on the driving task, despite being instructed to do so. Based on these findings, we would expect that, by adding feedback, participants would primarily focus their attention on the LCT in a dual-task driving environment. Thus, future studies are needed to compare single- and dualtask performance in the LCT with and without feedback.

\section{CONCLUSION}

To our knowledge, this study was the first to examine the effect of feedback in the LCT. The main finding was that providing participants with summary knowledge of results positively affected driving performance. Given the present findings, it might be important to include such feedback in future dual-task driving studies to encourage participants to prioritize the primary task. Research in our laboratory is currently investigating the effect of feedback on prioritization schemes in dual-task driving settings.

\section{ACKNOWLEDGMENTS}

This research was supported by the EU Marie Curie ITN Grant nr. 238833. We would like to thank Corinna Schönbeck and An Mai for their help with participant recruitment and data collection, and Richard Smolka, Ludger Blanke, Martin Finke and Andreas Volgmann for technical assistance. 


\section{REFERENCES}

Ammons, R. B. (1956). Effects of Knowledge of Performance: A Survey and Tentative Theoretical Formulation. The Journal of General Psychology, 54(2), 279 - 299.

Brumby, D. P., Salvucci, D. D., \& Howes, A. (2007). An empirical investigation into dual-task tradeoffs while driving and dialing. In D. Ramdny-Ellis \& D. Rachovides (Eds.), Volume 2 Proceedings of the 21st BCS HCI Group Conference (HCI 2007): Swindon, UK: BCS.

Chisholm, S. L., Caird, J. K., \& Lockhart, J. (2008). The effects of practice with MP3 players on driving performance. [doi: DOI: 10.1016/j.aap.2007.09.014]. Accident Analysis \& Prevention, 40(2), 704-713.

Donmez, B., Boyle, L. N., \& Lee, J. D. (2006). The Impact of Distraction Mitigation Strategies on Driving Performance. Human Factors: The Journal of the Human Factors and Ergonomics Society, 48(4), 785-804.

Hart, S., \& Staveland, L. (1988). Development of NASA-TLX (Task Load Index): Results of empirical and theoretical research. In P. Hancock \& N. Meshkati (Eds.), Human mental workload (pp. 139-183). Amsterdam: North Holland.

ISO (2010). ISO-26022: Road vehicles: Ergonomic aspects of transport information and control systems - Simulated lane change test to assess in-vehicle secondary task demand. International Organization of Standardization.

Latash, M. L., Scholz, J. P., \& Schöner, G. (2002). Motor control strategies revealed in the structure of motor variability. Exercise and Sport Sciences Reviews, 30(1), 26-31.

Levy, J., \& Pashler, H. (2008). Task prioritisation in multitasking during driving: opportunity to abort a concurrent task does not insulate braking responses from dual-task slowing. Applied Cognitive Psychology, 22(4), 507-525.

Mattes, S. (2003). The Lane-Change Task as a tool for driver distraction evaluation. In H. Rausch \& H. Bubb (Eds.), Quality of work products in enterprises of the future: Ergonomia Verlag.

Petzoldt, T., Bär, N., Ihle, C., \& Krems, J. F. (2011). Learning effects in the lane change task (LCT)-Evidence from two experimental studies. [doi: DOI: 10.1016/j.trf.2010.09.001]. Transportation Research Part F: Traffic Psychology and Behaviour, 14(1), 1-12.

Salmoni, A. W., Schmidt, R. A., \& Walter, C. B. (1984). Knowledge of results and motor learning: A review and critical reappraisal. [doi: DOI: 10.1037/0033-2909.95.3.355]. Psychological Bulletin, 95(3), 355-386.

Schmidt, R. A., Lange, C., \& Young, D. E. (1990). Optimizing summary knowledge of results for skill learning. [doi: DOI: 10.1016/0167-9457(90)90007-Z]. Human Movement Science, 9(3-5), 325-348.

Schmidt, R. A., \& Wulf, G. (1997). Continuous concurrent feedback degrades skill learning: Implications for training and simulation. Human Factors, 39, 509-525.

Shinar, D., Tractinsky, N., \& Compton, R. (2005). Effects of practice, age, and task demands, on interference from a phone task while driving. Accident Analysis \& Prevention, 37(2), 315-326.

Vollmeyer, R., \& Rheinberg, F. (2000). Does motivation affect performance via persistence? [doi: DOI: 10.1016/S0959-4752(99)00031-6]. Learning and Instruction, 10(4), 293-309.

Zijlstra, F., \& Van Doorn, T. (1985). The construction of a scale to measure perceived effort. Delft University of Technology, Delft, The Netherlands. 